\title{
Evolution des résidus de pesticides au cours des différents stades de la fabrication des aliments conservés ${ }^{(1)}$
}

\author{
par \\ J. P. FAUQUET \\ Docteur en Pharmacie, \\ Ancien Interne des Hôpitaux de Paris \\ et \\ G. LE MOAN \\ Professeur à la Faculté de Pharmacie de Paris, \\ Pharmacien des Hôpitaux
}

\section{INTRODUCTION}

L'utilisation massive des pesticides destinés à lutter contre les parasites des cultures et des récoltes est nécessaire pour protéger au maximum les ressources alimentaires d'origine végétale, mais introduit en contrepartie des risques nouveaux d'intoxications concernant directement les consommateurs.

Les hygiénistes et les toxicologues ont donc été amenés à s'intéresser aux problèmes posés par la présence et, plus récemment, par la persistance de résidus de pesticides dans les aliments, et à conseiller des mesures de sécurité par l'intermédiaire d'organismes internationaux (F.A.O., O.M.S.). En France, les différents aspects de la question ont été particulièrement bien étudiés par Truhaut [105], Le Moan [68] et Szakvary [98].

En comparaison des nombreuses études effectuées sur les résidus de pesticides sur les fruits et légumes frais après la récolte, relativement peu de travaux ont été effectués sur les mêmes aliments mis en conserve. Les conserves ont pourtant pris de plus en plus d'importance dans l'alimentation quotidienne par leur rapidité de préparation culinaire et leur facilité de stockage. Lors de leur fabrication, des règles strictes d'hygiène doivent assurer la régularité de la qualité afin de retrouver, après un délai plus ou moins pro-

(1) L'Alimentation et la Vie, 1969, 57, $\mathrm{n}^{\circ \mathrm{s}} 7-8-9$. 
longé, des denrées appétissantes se rapprochant le plus possible des préparations culinaires effectuées à partir de produits frais.

Pour évaluer l'importance des risques encourus par la présence de résidus de pesticides lors de la consommation des conserves, il est nécessaire de se poser différentes questions :

- Dans quelle mesure y a-t-il diminution des résidus lors du stockage des denrées après récolte et surtout au cours des différentes opérations de conserverie qui, a priori, doivent exercer un effet non négligeable ?

- Quels sont les effets du stockage des conserves sur les résidus ?

- Y a-t-il une interaction possible avec le métal des boîtes ?

- Les résidus de pesticides ou de leurs produits de transformation ont-ils un effet sur les caractères organoleptiques de produits en conserve ?

- Quelle est la toxicité des produits de dégradation des pesticides obtenus après les opérations de conserverie ?

Avant d'aborder l'étude de ces différents problèmes, il est nécessaire de délimiter notre sujet car la définition du mot "conserve » est très large et les procédés de préparation très divers. Dans le présent travail, il sera surtout question de denrées alimentaires mises en boîtes et conservées le plus souvent par stérilisation à la chaleur, c'est-à-dire par appertisation, et nous serons ainsi amenés à étudier l'influence des différents stades de préparation sur les résidus de pesticides :

- nettoyage, épluchage des légumes ou pelage des fruits, lavage ;

- blanchiment;

- mise en boîte et stérilisation.

Toutefois, au cours de notre exposé, nous pourrons être amenés à parler d'autres procédés de conservation : congélation, déshydratation, concentration, salage, etc.

Parmi les denrées susceptibles de contenir des résidus de pesticides et d'être mises en conserve, ce sont naturellement les fruits et légumes qui constitueront la matière principale de notre étude sans oublier leurs produits de transformation.

Toutefois, à côté d'une contamination directe, peut exister une contamination indirecte non négligeable de certains produits. Ainsi le lait provenant d'animaux alimentés avec des fourrages traités par les pesticides, est susceptible de contenir des résidus qui peuvent, par la suite, se concentrer dans le beurre. La pasteurisation de ces deux produits diminue-t-elle alors ces résidus ? 


\section{VI. - INFLUENCE DES OPERATIONS DE CONSERVATION SUR LES RESIDUS DE PESTICIDES DANS LE LAIT ET LES PRODUITS LAITIERS}

La contamination du lait résulte de l'emploi d'aérosols de pesticides au sein ou aux alentours des étables ou, surtout, de l'ingestion, par les vaches, d'aliments ou de fourrages pollués. Cette pollution résulte de l'usage inconsidéré des pesticides pour traiter les cultures et les récoltes et, en particulier, les pâturages. Les résidus de pesticides sont alors excrétés dans le lait et passent dans les produits laitiers.

De très nombreux travaux ont été consacrés à ce problème et ont montré que les risques de contamination étaient importants (Henderson [44]). Dans certains pays (U.S.A., Canada, U.R.S.S., Hollande), des contrôles analytiques systématiques ont été entrepris par l'industrie laitière et des doses limites tolérables sévères ont été établies pour les résidus (c'est le « zéro administratif », c'est-à-dire des teneurs inférieures à $2,5 \mathrm{ppm}$ rapportées à la matière grasse, soit $0,10 \mathrm{ppm}$ pour le lait, qui a constitué la base d'application du «Food, Drug and Cosmetic Act "). En France, par contre, il n'existe pas de contrôles systématiques et la législation se contente d'indiquer la liste des pesticides autorisés et leurs délais pour les traitements agricoles. L'insuffisance des méthodes prophylactiques a été bien mise en évidence par les travaux de Thieulin et coll. [100] qui ont montré que, sur 225 échantillons de lait et produits laitiers analysés, 60 à 92 p. 100 de ces échantillons étaient contaminés par des pesticides organochlorés (surtout $\mathrm{HCH}$ et dieldrine), les résidus maximum étant de 0,15 ppm pour le lait et 3,45 ppm pour le beurre.

Parmi les nombreux résultats accumulés sur les résidus de pesticides dans le lait et les produits laitiers, un nombre très limité d'informations a été recueilli sur les effets des méthodes de conservation sur l'importance de ces résidus.

En France, le Laboratoire coopératif d'analyses et de recherches [61] a évalué l'importance des résidus de DDT, DDE et $\mathrm{HCH}$ dans vingt échantillons de beurre (vrac, pasteurisés et laitiers) et a montré que le fait qu'un beurre soit pasteurisé ou non n'a pas d'influence sur la teneur en DDT ; celui-ci n'est pas détruit par la pasteurisation et c'est la qualité du lait initial qui compte seule.

Des études systématiques ont été effectuées par Langlois et coll. $[66,67]$ et Stemp et Liska [93] sur les effets des traitements de conservation sur les résidus d'insecticides organochlorés dans le lait et différents produits de transformation du lait. Les pesticides suivants ont été étudiés : DDT, lindane, endrine, dieldrine, heptachlore, télodrine et méthoxychlore. Ils ont été, soit incorporés dans la nourriture des vaches et excrétés dans le lait, soit ajoutés directement dans le lait avant fabrication des différents produits de transforma- 
tion et application des méthodes de conservation. Les produits suivants ont été préparés :

- Lait pasteurisé $\left(60^{\circ} \mathrm{C}\right.$ à $75^{\circ} \mathrm{C}$ pendant $\left.30 \mathrm{mn}\right)$ ou stérilisé ( $115^{\circ}$ C $\left.20 \mathrm{mn}\right)$.

- Lait condensé : condensation effectuée à chaud.

- Lait en poudre : fabrication par deux procédés : atomisation avec de l'air chaud ou tambour tournant chauffé.

- Beurre : fabriqué à partir de la crème, séparé du lait pasteurisé $\left(60^{\circ} \mathrm{C} 30 \mathrm{mn}\right)$ et stocké pendant 4 mois à basse température.

- Crème glacée : le mélange préalable nécessaire à la fabrication a été pasteurisé $\left(75^{\circ} \mathrm{C} 30 \mathrm{mn}\right)$, homogénéisé, refroidi à $+4^{\circ} \mathrm{C}$ puis transformé en crème glacée qui a été stockée à basse température pendant 4 mois.

\section{TABLEAU LI}

Effets des procédés de transformation et de conservation des produits laitiers sur les résidus de DDT et de lindane (d'après Langlois et coll. [66])

\begin{tabular}{|c|c|c|c|c|}
\hline \multirow[t]{2}{*}{ Traitements } & \multicolumn{2}{|c|}{$\begin{array}{l}\text { Résidus en ppm } \\
\text { (par rapport au poids) }\end{array}$} & \multicolumn{2}{|c|}{$\begin{array}{l}\text { Résidus en ppm } \\
\text { (par rapport à la } \\
\text { matière grasse) }\end{array}$} \\
\hline & $\left(^{*}\right) \mathrm{DDT}$ & *) Lindane & DDT & Lindane \\
\hline 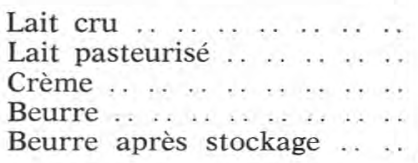 & $\begin{array}{r}0,90 \\
0,87 \\
10,70 \\
19,20 \\
21,80\end{array}$ & $\begin{array}{r}0,98 \\
0,80 \\
9,60 \\
20,00 \\
-\end{array}$ & $\begin{array}{l}26,47 \\
25,59 \\
28,89 \\
22,27 \\
25,29\end{array}$ & $\begin{array}{l}25,13 \\
20,51 \\
29,09 \\
23,60 \\
-\end{array}$ \\
\hline 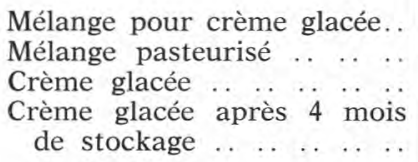 & $\begin{array}{l}0,318 \\
0,372 \\
0,298 \\
0,260\end{array}$ & $\begin{array}{l}0,190 \\
0,170 \\
0,170 \\
0,177\end{array}$ & $\begin{array}{l}2,93 \\
3,43 \\
2,65 \\
2,39\end{array}$ & $\begin{array}{l}1,67 \\
1,50 \\
1,50 \\
1,60\end{array}$ \\
\hline 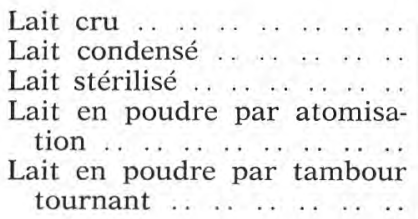 & $\begin{array}{r}0,91 \\
1,78 \\
1,94 \\
2,75 \\
12,20\end{array}$ & $\begin{array}{l}1,00 \\
2,00 \\
1,94 \\
1,22 \\
2,71\end{array}$ & $\begin{array}{r}26,00 \\
23,05 \\
25,12 \\
9,85 \\
\\
43,43\end{array}$ & $\begin{array}{r}25,00 \\
26,42 \\
25,63 \\
4,38 \\
9,27\end{array}$ \\
\hline
\end{tabular}

(*) Lors de la fabrication du beurre et des différentes sortes de laits, le DDT a été ajouté au lait tandis que le lindane a été ajouté au fourrage servant à la nourriture des vaches et a été excrété dans le lait. Dans le cas des crèmes glacées, le DDT et le lindane ont été ajoutés au mélange initial pour crème glacée. 
Les résultats obtenus par ces différentes opérations sur les résidus de pesticides, sont résumés dans les tableaux LI, LII, LIII. D'après les chiffres indiqués, nous voyons que les résidus de DDT et lindane sont stables durant les différentes opérations de conservation. Les résidus sont les mêmes dans les produits finis et dans le lait cru de départ si l'on exprime les résultats en fonction de la matière grasse. La seule perte significative se produit dans le cas du lait en poudre par atomisation pour le DDT et le lindane et, dans le cas du lait en poudre, par le procédé du tambour tournant pour

\section{TABLEAU LII}

Effets des procédés de transformation et de conservation des produits laitiers sur les résidus de dieldrine, d'endrine, d'heptachlore et d'heptachlorépoxyde (d'après Langlois et coll. [67])

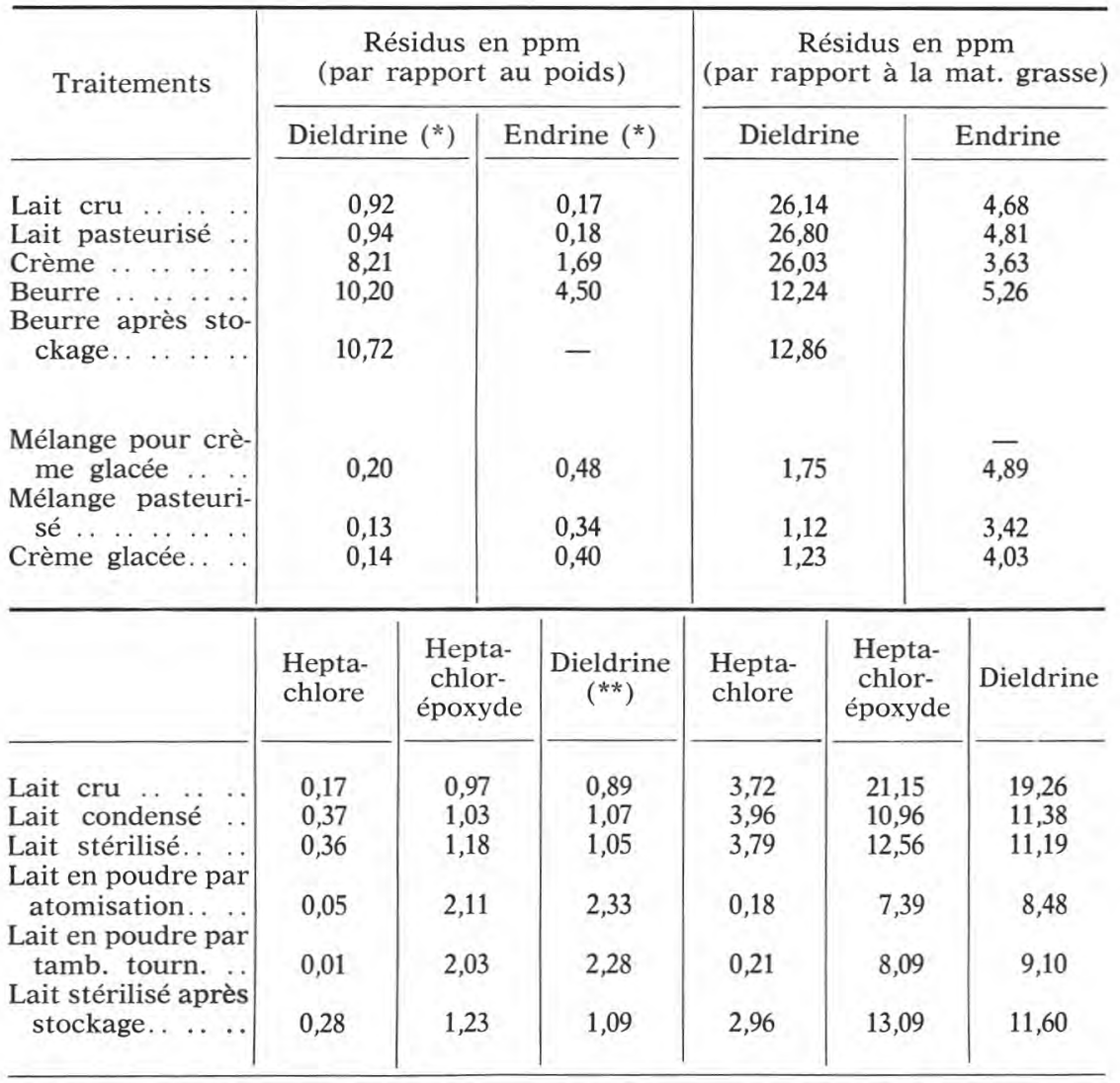

(*) La dieldrine a été ajoutée au lait tandis que l'endrine a été ajoutée au fourrage servant à la nourriture des vaches et a été excrétée dans le lait.

$\left.{ }^{* *}\right)$ L'heptachlore et la dieldrine ont été ajoutés au fourrage des vaches. 


\section{TABLEAU LIII}

Effets des procédés de transformation et de conservation des produits laitiers sur les résidus de télodrine et de méthoxychlore (d'après Stemp et Liska [93])

\begin{tabular}{|c|c|c|c|c|}
\hline \multirow{2}{*}{ Traitements } & \multicolumn{2}{|c|}{$\begin{array}{l}\text { Résidus en ppm } \\
\text { (par rapport au poids) }\end{array}$} & \multicolumn{2}{|c|}{$\begin{array}{l}\text { Résidus en ppm } \\
\text { (par rapport à la matièr } \\
\text { grasse) }\end{array}$} \\
\hline & $\begin{array}{l}\text { Télodrine } \\
\qquad(*)\end{array}$ & $\begin{array}{l}\text { Méthoxy- } \\
\text { chlore (**) }\end{array}$ & Télodrine & $\begin{array}{l}\text { Méthoxy- } \\
\text { chlore }\end{array}$ \\
\hline Lait cru & 0,81 & 1,02 & 18,3 & 23,1 \\
\hline Lait condensé ....... & 1,72 & 1,89 & $-17,8$ & 21,1 \\
\hline Lait en poudre par atomi- & & & & \\
\hline $\begin{array}{l}\text { sation } \\
\text { Lait en poudre par tam- }\end{array}$ & 4,72 & 6,76 & 16,3 & 22,7 \\
\hline $\begin{array}{l}\text { Lait en poudre par tam- } \\
\text { bour tournant } \ldots . . .\end{array}$ & 4,80 & 6,78 & 16,5 & 23,3 \\
\hline Lait stérilisé $\ldots \ldots \ldots \ldots$ & 0,92 & 1,70 & 10,2 & 18,6 \\
\hline
\end{tabular}

(*) La télodrine a été ajoutée au fourrage destiné à la nourriture des vaches et a été excrétée dans le lait.

${ }^{* *}$ Le méthoxychlore a été ajouté directement au lait.

le lindane, si on se réfère aux résultats exprimés en fonction de la matière grasse.

En ce qui concerne endrine, dieldrine et heptachlorépoxyde (métabolite de l'heptachlore), en exprimant les résidus par rapport à la matière grasse, on note une perte d'heptachlorépoxyde et de dieldrine dans le lait condensé alors que l'heptachlore lui-même et l'endrine ne sont pas affectés. Une diminution de l'heptachlore, de l'heptachlorépoxyde et de la dieldrine est observée pour le lait en poudre, mais aucun changement (excepté une diminution de l'heptachlore) ne se produit dans le cas de la crème glacée. Il n'y a pas de modification des résidus après stockage du beurre, de la crème glacée et du lait stérilisé. La diminution enregistrée pour la dieldrine dans le cas du beurre (résultats exprimés en fonction de la matière grasse) est due uniquement à une perte au cours de l'écrémage.

Dans le cas de la télodrine et du méthoxychlore, il n'y a pratiquement pas de diminution des résidus pour le lait condensé. On enregistre pour la télodrine une perte de 10 à 20 p. 100 dans le lait en poudre et de 40 à 50 p. 100 dans le lait stérilisé (légère diminution du méthoxychlore dans le lait stérilisé).

Ces résultats sont confirmés par ceux obtenus par Kiermeier et coll. [51] qui ont étudié les résidus de DDT, de méthoxychlore, de dieldrine, d'heptachlorépoxyde et de lindane dans le lait en poudre après addition volontaire de ces pesticides dans le lait cru. Seuls les 
résidus d'heptachlorépoxyde et de lindane sont diminués respectivement de 27 et 41 p. 100 environ après lyophilisation du lait.

En conclusion, les résidus de pesticides, tout au moins en ce qui concerne ceux retrouvés le plus couramment dans le lait, semblent résister facilement aux effets des méthodes de conservation. La présence ou l'absence de résidus dans les produits laitiers dépend donc seulement de la qualité du lait initial et, par conséquent, des mesures de prévention prises pour éviter la contamination et pour limiter l'emploi des pesticides à des périodes bien définies, Les risques encourus par le consommateur ne sont pas négligeables et il ne faut pas oublier, à ce propos, que le lait constitue l'aliment de base du nourrisson. Toutefois, des résultats rassurants sont rapportés par Henderson [44]. Aux U.S.A., l'industrie laitière a fait un gros effort pour la réduction des résidus dans le lait et les produits laitiers. Des laboratoires d'analyses ont été créés et, pendant une période de deux ans, 31548 échantillons de lait et de produits laitiers ont été analysés et les résultats de ces recherches transmis au Comité des Industries laitières. Le rapport d'ensemble indique que les taux résiduels groupés de DDT, DDE, DDD et de méthoxychlore restent dans la plupart des cas, très inférieurs à $0,1 \mathrm{ppm}$ rapporté au lait total ou à 2,5 ppm rapportés aux matières grasses.

\section{B ibliographie}

[44] Henderson (J. L.) (1965). - Residue reviews, 8, 74.

[51] Kiermeier (F.), Rudolphi (M.) et Wildbrett (G.) (1967). - Z. Lebensmitt. Untersuch. Forsch., 132 (2), 78.

[61] LabORATOIRE COOPÉRATIF D'ANALySES ET DE RECHERCHES. - Bulletin d'Information, 1966, mars-avril, $\mathrm{n}^{\circ} 47,6$.

[66] Langlois (B. E.), Liska (B. J.) et Hill (D. L.) (1964). - J. Milk Food Technol., 27, 264.

[67] Langlois (B. E.), Liska (B. J.) et Hill (D. L.) (1965). - J. Milk Food Technol., 28 (1), 9.

[68] Le Monn (G.). - Conférence donnée au Palais de la Découverte le 14 mars 1964. .............

[93] Stemp (A. R.) et Liska (B. J.). (1966) - J. Dairy Sci., 49 (8), 1006.

[98] Szakvary (A.) (1965). - Travail du Centre de Recherches Foch, Foulon édit,, Paris.

[100] Thieulin (G.), Pantaleon (J.), Richou (L.) et Cumont (G.). - Bull. Acad. Nat. Méd., 1966, 150 (24-25), 478 ; Le Lait, 1967, $\mathrm{n}^{\circ} 461-462$.

[105] Truhaut (R.) (1966). - Ann. Hyg. L. Fr., 2 (5), 15. 